\title{
Solid-Pseudopapillary Tumor of the Pancreas: Our Experience in Bangladesh
}

\author{
ZR KHAN ${ }^{\mathrm{a}}$, BC DAS ${ }^{\mathrm{b}}, \mathrm{M} \mathrm{CHOWDHURYc}^{\mathrm{c}}$, MS UDDIN ${ }^{\mathrm{d}}$, A TAHER $^{\mathrm{e}}$, SM HOSSAIN $^{\mathrm{f}}$
}

\begin{abstract}
:
Background: Solid-pseudopapillary tumors of the pancreas (SPTPs) have been reported as rare lesions with "low malignant potential” occurring mainly in young women. This study was designed to understand clinicopathological characteristics of the disease, management strategy and outcome of this rare disease in Bangladesh.
\end{abstract}

Methods: A retrospective review from January 2001 to December 2009 was performed. Clinicopathological, peroperative, postoperative and survival data were obtained from record file. Our cases were discussed in the light of published literature.

Results: During this period, 31 patients were diagnosed as having SPTPs (6.9\%). Twenty-four (77.4\%) females and seven (22.6\%) males were identified, with a median age of 24 years (range, 14-44). The median size of the lesions was

\section{Introduction:}

Solid pseudopapillary tumor of the pancreas (SPTP) is a rare pathologic entity with low malignant potential ${ }^{1}$ affecting primarily young women ${ }^{2}$. Recently there has been a steady increase in the number of SPTP of the pancreas. Despite the increase in recognition, the

a. Dr. Zulfiqur Rahman Khan, FCPS, FRCS, Department of Surgery, Bangabhandhu Shiekh Mujib Medical University, Shahbag, Dhaka

b. Dr. Bidhan C. Das, FCPS, PhD, Department of Surgery, Bangabhandhu Shiekh Mujib Medical University, Shahbag, Dhaka

c. Dr. Mohsen Chowdhury, FCPS, Department of Surgery, Bangabhandhu Shiekh Mujib Medical University, Shahbag, Dhaka

d. Dr. Mohammad Saief Uddin, MRCS, Department of Surgery, Bangabhandhu Shiekh Mujib Medical University, Shahbag, Dhaka

e. Dr. Abu Taher, MS, Department of Surgery, Bangabhandhu Shiekh Mujib Medical University, Shahbag, Dhaka

f. Dr. Syed Mokarrom Hossain, MBBS Department of Surgery, Bangabhandhu Shiekh Mujib Medical University, Shahbag, Dhaka

Address of Correspondence: Prof. Zulfiqur Rahman Khan, FCPS, FRCS, Professor of Surgery, Department of Surgery, Room no-909, Block C, Bangabhandhu Shiekh Mujib Medical University, Shahbag, Dhaka

Received: 23 May, 2010

Accepted: 22 July 2010
$7.0 \mathrm{~cm}$ (range, $4.8-18$ ). More than $90 \%$ presented with vague abdominal pain and lump. Twenty five patients had their primary tumors within the head of pancreas, the rest were in body and tail region. A total of 30 patients presented with local disease and underwent complete resection. One patient was found to have a very large tumor in the head with vascular invasion and underwent debulking of the tumor; which clinically reappear 1 year after debulking but still alive with episodic attacks of abdominal pain. All patients are surviving till date at a follow-up of 2 months to 9 years. Conclusions: SPTP occurs predominantly in women although it can occur in men. Young and middle aged groups are affected. Complete resection or debulking of locally invasive tumor is associated with long-term survival.

(J Bangladesh Coll Phys Surg 2011; 29: 138-142)

pathogenesis and apparent therapeutic algorithm remain unclear. Because of vague initial symptoms the tumor becomes very large on presentation. It is often seemed to be a locally invasive tumor on clinical and imaging evaluation and label the tumor as an irresectable one. This study was designed to examine the clinicopathological characteristics of the disease, management strategy and outcome by examining our institutional experience.

Patients and Methods:

We reviewed 451 patients with pancreatic malignancy those were admitted to Bangabandhu Sheikh Mujib Medical University (BSMMU), Bangladesh and other hospitals in Dhaka city, and underwent pancreatic resection during 2001 to 2009. Clinico-pathological, peroperative, and postoperative data were obtained from record file. The follow up records were obtained from out patient department follow up file and personal contact with the patient. This study was designed to understand clinical behavior, management plan and outcome of these rare tumors in Bangladesh

\section{Results:}

Out of the 451 patients with pancreatic malignancy histopathological report diagnosed 31 as having SPTP 
(6.9) (Table-I). There were 24 females and 7 males with a median age of 24 years (range, 14-44). The predominant presenting symptom was abdominal pain and lump ( $\mathrm{n}=28,90.3 \%)$; two patients had jaundice (6.7 $\%$ ) and one patient was asymptomatic (3.0\%). The tumor markers levels of all patients (CA19-9 \& CEA) were within normal limits. The median size of the lesions was $7.0 \mathrm{~cm}$ (range, 4.5-18) (Table -II).

\section{Table-I}

\begin{tabular}{|c|c|c|}
\hline Sl no & Types of pancreatic tumor & Number (\%) \\
\hline 1 & Adenocarcinoma & 418 (93.7) \\
\hline 2 & $\begin{array}{l}\text { Solid pseudopapillary tumor of } \\
\text { pancreas (SPTP) }\end{array}$ & $31(6.9)$ \\
\hline 3 & Non Beta cell tumor & $1(0.2)$ \\
\hline \multirow[t]{2}{*}{4} & Non Hodgkin tumor & $1(0.2)$ \\
\hline & Total & 451 (100) \\
\hline
\end{tabular}

Table-II

Clinical data of patients with solid pseudopapillary tumor of pancreas

\begin{tabular}{ll} 
Total patient & 31 \\
\hline Male : Female & $7: 24$ \\
Median Age (years) & 24 (range: 14 to 44) \\
Clinical Presentation & 25 \\
Abdominal pain and lump & 28 (90.3\%) \\
Jaundice & $02(6.7 \%)$ \\
Incidental & 01 (3.0\%) \\
Tumor size (Median) & $7.0 \mathrm{~cm}$ (range: 4.5 to 18) \\
Tumor location & 25 (80.6\%) \\
$\quad$ Head & 02 (6.5\%) \\
$\quad$ Body & $04(12.9 \%$ \\
\hline
\end{tabular}

Twenty five patients had their primary tumors within the head, 2 in the body and 4 in the tail of the pancreas (Fig-1) Preoperatively, CT guided FNAC of the lump in initial few cases were inconclusive; therefore FNAC was abandoned in later cases. Laparotomy was planned for resection of the tumor; however triple bypass and tissue biopsy from the lesion was taken into consideration in case of irresectable tumor. A total of 30 patients presented with local disease underwent resection. Twenty four patients underwent pancreaticoduodenectomy (Fig 2a), four patients underwent a distal pancreatectomy (Fig. 2b) and two patients underwent enucleation of the tumor. One patient was found to have a very large tumor in the head with vascular invasion (Fig.-3) and underwent debulking of the tumor. Postoperatively, 23 patients (74.1\%) had an uneventful course; rest 8 patients (25.9\%) had minor complications; wound infection and wound dehiscence in 2, atelectasis in 1 , thrombophlebitis in 2 patients.

Tumors were generally large, varied from tan to yellow, and showed irregular cystic cavities lined by soft, friable tissue. Foci of hemorrhage were common. Some examples also demonstrated firm, fibrotic regions within the tumor. Most cases appeared to be grossly well circumscribed or even partially encapsulated. The microscopic appearance of all the cases demonstrated the characteristic microscopic features of SPTP. The solid areas were composed of monotonous polygonal epithelioid cells, often with minimal intervening stroma, accompanied by innumerable capillary-sized vessels. Some areas showed more extensive stromal fibrosis, with round aggregates of perivascular hyalinized stroma imparting a cylindromatous appearance. In the pseudopapillary regions, the cells located away from the small vessels appeared to have dropped away, leaving an irregular cuff of cells surrounding each vascular core. There was evidence of cellular degeneration, including aggregates of foamy histocytes, cholesterol clefts, and cytoplasmic vacuolization. Clusters of cells demonstrated large eosinophilic cytoplasmic globules. The nuclei were generally uniform and round to oval, with longitudinal grooves. The microscopic interface between the tumors and the adjacent pancreas commonly showed an infiltrative growth pattern, with islands of non-neoplastic pancreatic parenchyma entrapped within the tumor and nests of tumor cells extending into the adjacent pancreas (Fig-4). 

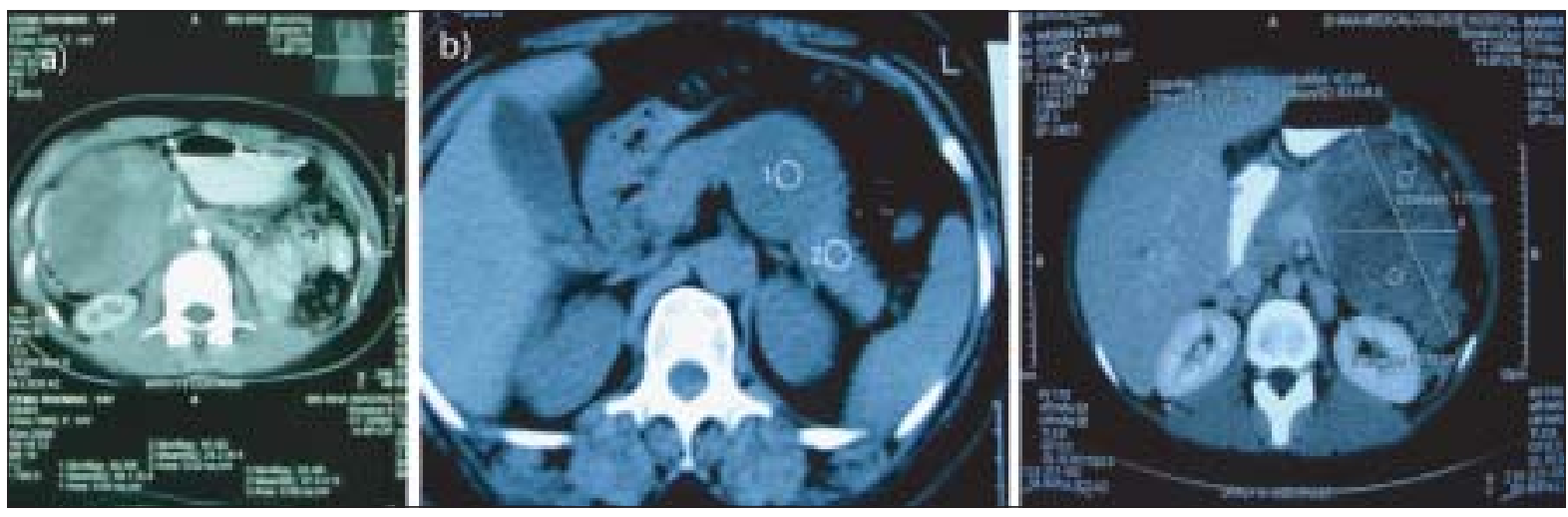

Fig. 1: Location of the tumor a) Head of the pancreas, b) Body of the pancreas, c) body and tail of the pancreas

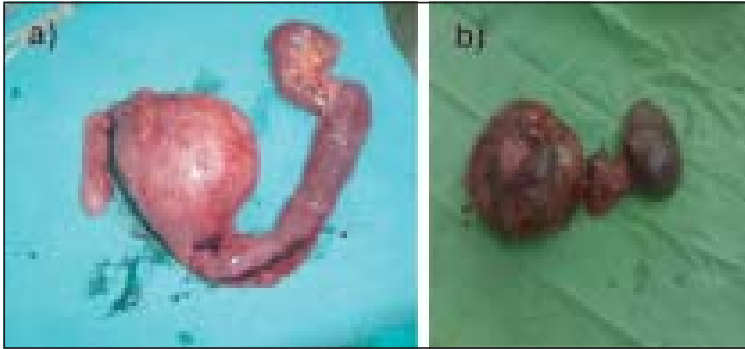

Fig.-2: Specimen of a) pancreatoduodenectomy and b) distal pancreatectomy

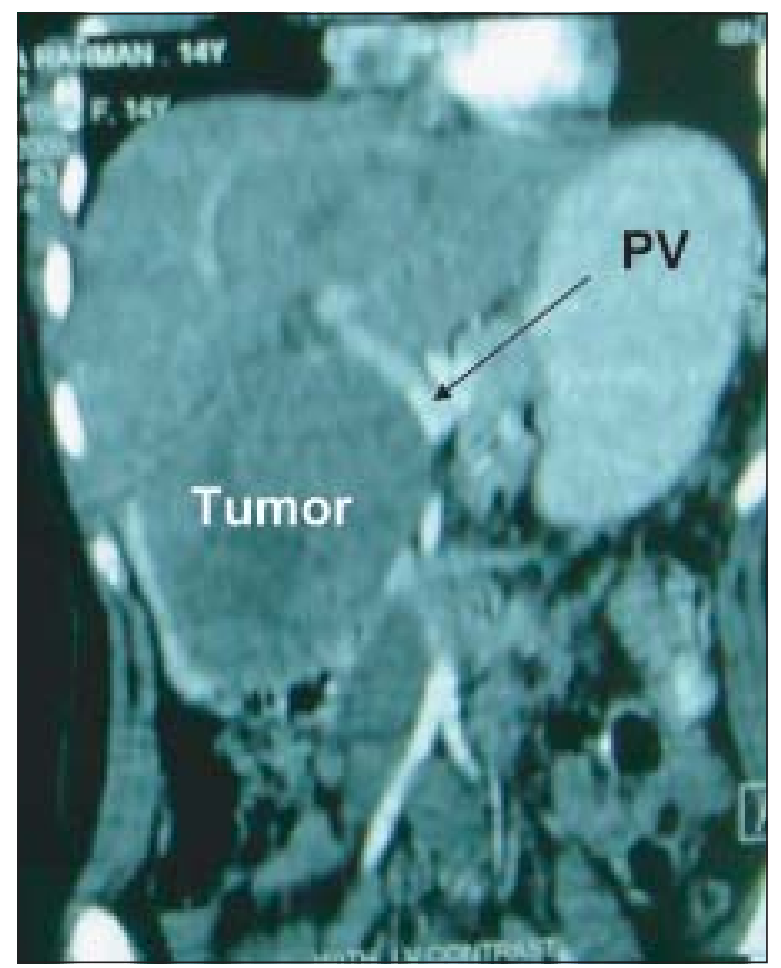

Fig.-3: Tumor compressing the portal vein (PV)

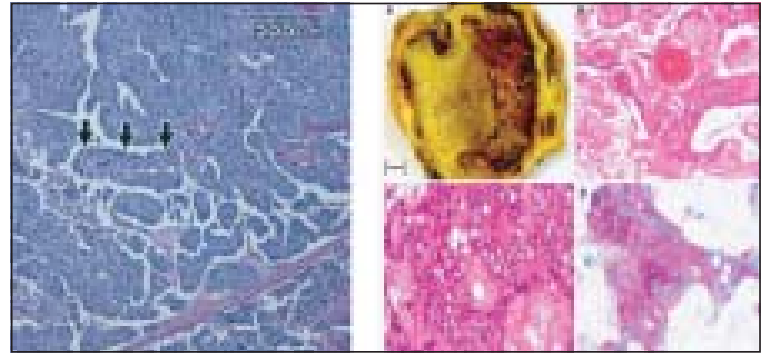

Fig.-4: Histopathology of Solid Pseudopapillary Tumor of Pancreas

At a follow-up of 2 months to 9 years, all patients are surviving till date; no patients had any evidence of recurrence except one who underwent debulking of the tumor. This patient's tumor clinically reappears 1 year after debulking, and the patient is still alive with episodic attack of abdominal pain

\section{Discussion:}

SPTP is a very rare variety of pancreatic tumor. It was first described in 1959 by Frantz ${ }^{3}$. World Health Organization named this tumor as SPTP in 1996. ${ }^{4}$ Before that it has been described by many other terms, such as papillary epithelial neoplasm, solid and cystic acinar cell tumor, papillary cystic neoplasm, papillary cystic carcinoma, solid and cystic tumor, low-grade papillary tumor, and Frantz's tumor. It has also been misdiagnosed as adenocarcinoma, islet cell tumors, cystadenomas, papillary cystadenocarcinoma, or cystadenocarcinoma.

A total of 719 cases of SPTP of the pancreas have been reported in the literature since it was first described in $1959^{5,6}$. There has been an increasing incidence of this 
entity in recent years. A possible explanation of increasing incidence is greater awareness of this disease, as well as better understanding of pancreatic pathology with the 1996 new classifications of pancreatic neoplasms from the World Health Organization ${ }^{7}$.

SPTPs of the pancreas have been diagnosed most commonly in young women. The series has demonstrated the incidence of male patients diagnosed with this disease similar to the series of Memorial SloanKettering Cancer Center, New York ${ }^{8}$. A majority of reports have found the greatest incidence of SPTP of the pancreas in the third decade of life; however, our patients presented with a median age of 24 years, which is similar with two large single institutional series and in the total number of cases reported (median age of 26 years $)^{9,10}$. However Robert $C$ et al reported SPTP with a median age of 39 years.

More than 90\% patients in this series presented with vague abdominal pain and abdominal lump. Symptoms, when they occur, are often vague and nonspecific, leading to a delay in diagnosis. As a result of these subtle symptoms, tumor presentation can be quite large. But size of the lesion is not a predictor of unresectability, as seen in this series with maximum size of $18 \mathrm{~cm}$ and in others, with lesions 20 to $30 \mathrm{~cm}$ in size still being resectable ${ }^{11,12}$. These lesions rarely invade contiguous structures or occlude the bile ducts as occurred in only two of our patients presenting with jaundice. Vascular invasion, althoughuncommon, does occur, as evidenced by one of our patient who presented with portal vein encasement.

The cell of origin of the solid -pseudopapillary tumour is uncertain and has not yet been clarified. It is a low malignant potential tumor, complete resection or enucleation whenever possible and even debulking of the tumor provide a good survival time. In our series all patient survived 2 month to 9 years without recurrence except one patient who underwent debulking of the tumor still surviving one year after operation.

Metastatic disease does occur with SPTPs, with 20 previously reported cases ${ }^{13,14}$. The most common site of distant disease is the liver; very rare cases of lymph node and peritoneal spread have been reported. Disseminated disease is also not a negative predictor of survival. Long-term survival, 7 to 10 years, has been reported in patients undergoing complete resection, but it is more important to note that it has also been reported in patients with residual disease $\mathrm{11}^{11,14}$. In Robert $\mathrm{C}$ et al series, two patients with liver metastasis had significant overall survival, with one alive at 11 years and the other alive with liver recurrence at 4 years.

In most studies of the clinical outcome of SPTPs, no pathologic factors predictive of prognosis have been identified. In the largest clinicopathological study to date (published only in abstract form), pathologic factors including mitotic rate, nuclear pleomorphism, and vascular invasion were not found to correlate with prognosis $^{15}$.

Adjuvant therapy has been used only in a small number of patients as published in the literature. Many different regimens of chemotherapy have been used without any demonstration of response. One patient of a series ${ }^{\mathbf{8}}$ was treated with complete cycles of 5-fluorouracil, doxorubicin, and streptozocin and interferon, cisplatin, and topotecan without any response to the primary lesion. Radiotherapy has been used infrequently. Only one case report indicates significant success in a locally advanced lesion involving the porta hepatis; it responded to 4000 cGy over 6 weeks with a 3 -year follow-up ${ }^{16}$. Other reports have also looked at estrogen receptor status and have found no indication that over expression exists in these lesions ${ }^{10}$.

\section{Summary:}

Our experience with SPTP showed young adult female are commonly sufferer. Because of vague initial symptoms, tumor becomes large at presentation. Aggressive treatment with attempts made for complete resection, even if this requires metastasectomy can provide long-term survival for SPTP. Adjuvant therapy did not show any improvement of survival in such patients as per literature review.

\section{References:}

1. Compagno J, Oertel JE, Kemzar M. Solid and papillary epithelial neoplasm of the pancreas, probably of small-duct origin: a clinico-pathological study of 52 cases [Abstract]. Lab Invest 1979; 40: 248-49.

2. Oertel JE, Mendelsohn G, Compagno J. Solid and papillary epithelial neoplasms of the pancreas. In: Pancreatic Tumors in Children. Dordrecht, the Netherlands: Martinus Nijhoff, 1982: 167-71.

3. Frantz V. K. Tumors of the pancreas. In : Atlas of tumor pathology. Washington DC : Armed Forces Institute of Pathology, 1959, pp 32-33 
4. Kloppel G, Solcia E, Longnecker DS. Histological typing of tumors of the exocrine pancreas. 2nd ed. (WHO international histological classification of tumors). Berlin, Heidelberg, New York: Springer; 1996.

5. Fried P, Cooper J, Balthazar E, Fazzini E, Newall J. A role for radiotherapy in the treatment of solid and papillary neoplasms of the pancreas. Cancer 1985; 56: 2783-5

6. Mao C, Guvendi M, Domenico DR, Kim K, Thomford NR, Howard JM. Papillary cystic and solid tumors of the pancreas: a pancreatic embryonic tumor? Studies of three cases and cumulative review of the world's literature. Surgery 1995; 118: 821-8.

7. Kloppel G, Solcia E, Longnecker DS, Capella C, Sobin LH. Histological Typing of Tumors of the Exocrine Pancreas. New York: Springer, 1996.

8. Robert C. G. Martin, MD, David S. Klimstra, MD, Murray F. Brennan, MD and Kevin C. Conlon, MD SolidPseudopapillary Tumor of the Pancreas: A Surgical Enigma? Annals of Surgical Oncology 9:35-40 (2002)

9. Pettinato G, Manivel JC, Ravetto C, et al. Papillary cystic tumor of the pancreas. A clinicopathologic study of 20 cases with cytologic, immunohistochemical, ultrastructural, and flow cytometric observations, and a review of the literature (published erratum appears in Am J Clin Pathol 1993;99:764). Am J Clin Pathol 1992; 98: 478-88
10. Lam KY, Lo CY, Fan ST. Pancreatic solid-cystic-papillary tumor: clinicopathologic features in eight patients from Hong Kong and review of the literature. World J Surg 1999; 23: 1045-50.

11. Zinner MJ, Shurbaji MS, Cameron JL. Solid and papillary epithelial neoplasms of the pancreas. Surgery 1990; 108: 47580.

10. Kingsnorth AN, Galloway SW, Lewis-Jones H, Nash JR, Smith PA. Papillary cystic neoplasm of the pancreas: presentation and natural history in two cases. Gut 1992; 33: 421-3

12. Sclafani LM, Reuter VE, Coit DG, Brennan MF. The malignant nature of papillary and cystic neoplasm of the pancreas. Cancer 1991; 68: 153-8

14. Nishihara K, Nagoshi M, Tsuneyoshi M, Yamaguchi K, Hayashi I. Papillary cystic tumors of the pancreas. Assessment of their malignant potential. Cancer 1993; 71: 82-92

15. Adair CF, Wenig BM, Heffess CS. Solid and papillary cystic carcinoma of the pancreas: a tumor of low malignant potential (abstract). Int J Surg Pathol 1995; 2: 326

16 Fried P, Cooper J, Balthazar E, Fazzini E, Newall J. A role for radiotherapy in the treatment of solid and papillary neoplasms of the pancreas. Cancer 1985; 56: 2783-5 